\title{
Home Automation Systems - A Study
}

\author{
Satish \\ Palaniappan \\ CSE, SSN College \\ of Engineering, \\ Anna University, \\ Chennai, India
}

\author{
Naveen \\ Hariharan \\ CSE, SSN College \\ of Engineering, \\ Anna University, \\ Chennai, India
}

\author{
Naren T Kesh \\ CSE, SSN College \\ of Engineering, \\ Anna University, \\ Chennai, India
}

\author{
Vidhyalakshimi S \\ CSE, SSN College \\ of Engineering, \\ Anna University, \\ Chennai, India
}

\author{
Angel Deborah S \\ Assistant Prof., \\ CSE, \\ SSN College of \\ Engineering, \\ Anna University, \\ Chennai, India
}

\begin{abstract}
With the increase in consumption of energy and population, there is a grave need to conserve energy in every way possible. The inability to access and control the appliances from remote locations is one of the major reasons for energy loss. A web or an android application is used by the users to give instructions to these systems. This system can make use of a host of communication methods such as Wi-Fi, GSM, Bluetooth, ZigBee. Different controlling devices and configurations can be found in existing systems. Such systems have been found already in many places for a wide variety of applications. This paper presents a survey of all such systems.
\end{abstract}

\section{General Terms}

Embedded Systems, Remote access Systems, Mobile Applications, Web Applications and Home Automation System

\section{Keywords}

Energy Conservation, Raspberry Pi, Portability, Physically Challenged, Electrical and Electronic Devices, Home Automation.

\section{INTRODUCTION}

Electronic and Electrical environment with respect to this context is any environment which consists of appliances such as fans, television sets, air conditioners, motors, heater, lighting systems, etc. A remotely accessible environment is an environment in which each appliance can be remotely accessed and controlled using software as an interface, which includes an Android application and a Web application. Such remotely accessible systems are already available in the market, but have a number of drawbacks as well. This paper aims to perform a survey of all the existing such systems and compare the available features.

The paper will also compare and contrast all the systems and look at their various features and disadvantages. A wide variety of options are available for the home automation systems. All of these will be examined at length.

\section{METHODOLOGIES}

\subsection{GSM based Home Automation System}

The system proposed in [1] provides 3 means to control the home: the GSM network, the Internet and through speech. The real time monitoring has been an important feature that can be used in the home automation systems. As a change in the status of the devices occurs, the user can be informed in real time. The user commands are transferred to a server which is usually done by a PC. The server processes the user commands and sends them to the relevant units. This can help control the appliances. GSM is used as a communication medium to help establish connection in places where there may not be proper internet connectivity. The server uses AT commands to communicate with the GSM modem. The mobile interface is developed using J2ME. The server has 4 engines running - the web server, database, main control program and speech recognition program. The system can be controlled using SMS. It can send confirmation messages. Speech processing is done with a dynamic time wrapping algorithm. The voice activation has been tested and found to be too impractical. As a more stable alternative, the voice input can be activated through a wireless unit the user carries along in the house. Each application node has four parts - the transmitter, receiver, I/O device and a microcontroller. The main control program in the server takes status information from the devices' transceiver in real time.

The system makes use of a PIC16F887 microcontroller for home appliances control [2]. It makes use of GSM for control of the appliances. This is an SMS based system. GSM has been used due to its high availability, coverage and security. The control of home appliances is done primarily through SMS codes. AT commands can be sent through the GSM network and this controls the home devices. Messages are sent by the device to the user through SMS as well. This system can however incur additional costs for the SMS. There is no UI that the user can use to control the device. This system has the drawback of not being able to program the devices. Also SMS depends on the networks and there is a possibility of delayed delivery. The system does not does not have any state information related to the devices and expects the user to keep track of it.

The system [3] is described as an M2M system. It uses GSM for communication. GSM offers options for M2M which include Dual Tone Multi Frequency (DTMF), SMS and

General Packet Radio Service (GPRS). This system chooses to use the SMS along with AT (attention) commands. It has a $\mathrm{PC}$ as a centre of commands. A GSM dial-up and communication system is embedded in the PC. Visual $\mathrm{C}++$ is used for implementation. The PC decodes the received messages via SMS and performs the commands required. It is a system that can be programmed for the required application as per requirements. The system also has the ability to control mechanical appliances, through sensors that convert electrical to mechanical signals. However, this system is not designed to provide feedback to the user. The system is PC centric and requires the $\mathrm{PC}$ to be on all the time. It cannot be used as a real time control system.

The home server is built upon a SMS/GPRS mobile cell module and a microcontroller ${ }^{[4]}$. This allows the user to monitor and control any appliances at home using any Java enabled cell phone. The paper presents the design and 
implementation of AT modem driver, text based command processing software and power failure resilient output from the microcontroller. The mobile phones are used to provide a user friendly interface. They also serve to send commands and receive feedback from the system as SMS strings. The hardware components include an Atmel microcontroller, which is connected with a RS232 serial port. It also has EEPROM memory to ensure that the relevant details are stored. A password based authentication system has to be used. The text messages sent will contain the password which is used to ensure the message is sent from a valid source. The main drawback of this system is that it relies heavily on the SMS, which is not very fast and dependable. There can be delays in delivery. Also security of the system is compromised since passwords are sent freely over the network.
A system based on GSM network via SMS [5] is used to control the home appliances as shown in figure 1. An Arduino board is the controller used to interface the appliances. It uses certain peripheral drivers and relays to achieve this interfacing. The smart phone is the user interface device. The system uses the 'App Inventor' visual programming tool to develop the interface and other tools to deploy the app. The app generates SMS messages based on the user commands and sends it to the GSM modem attached to the Arduino. This allows the user to control the home appliances. The system suffers from the same drawbacks of cost and reliability of SMS. Also the interface is pre programmed and cannot be customized based on devices.

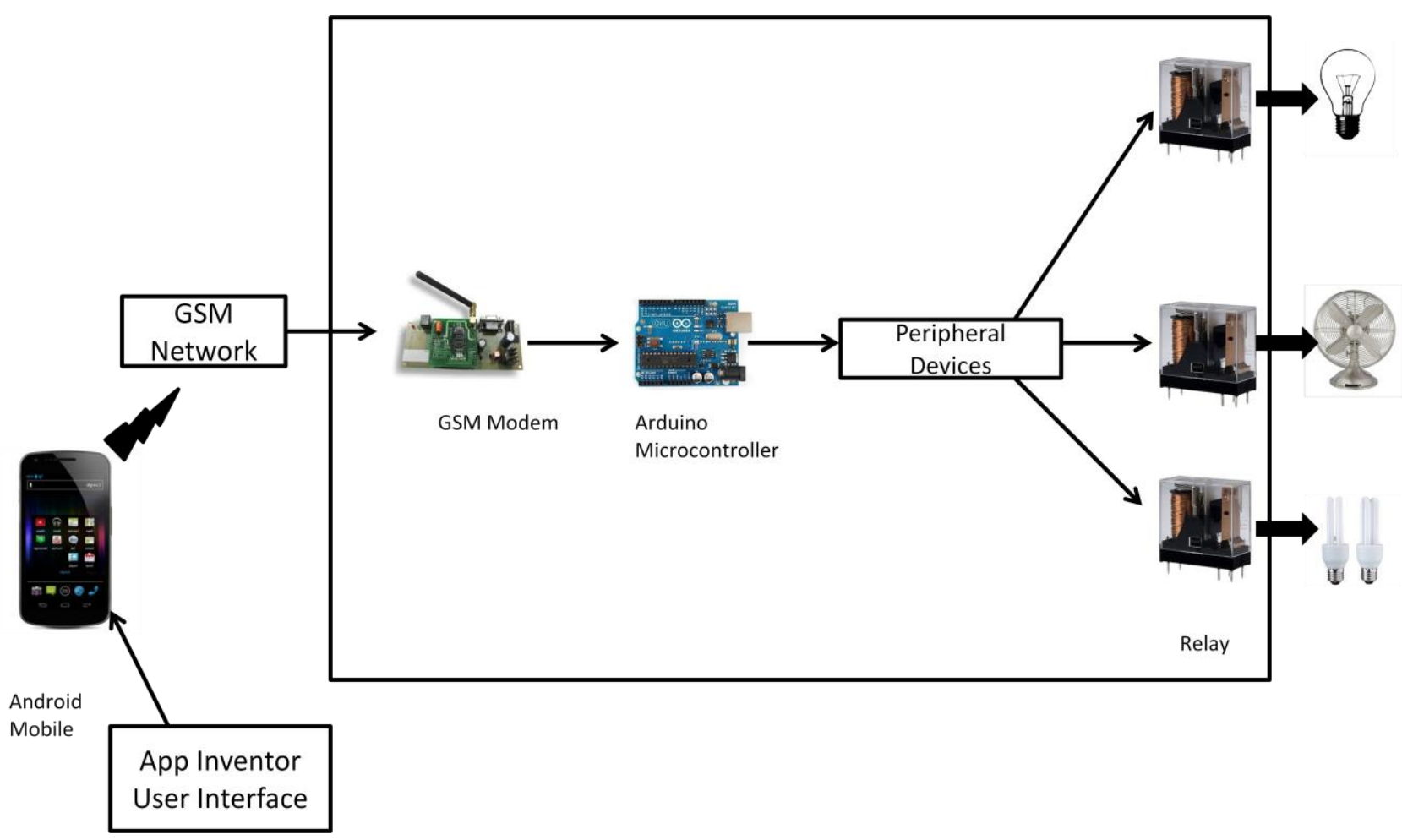

Figure 1 GSM based HAS

A voice control system is proposed ${ }^{[6]}$ that will enable the elderly and disabled to control appliances remotely. The primary communication means is through GSM. An Android mobile phone is used to get the voice commands and converts them into text. This is sent via SMS to another phone through the GSM network. This other phone uses Bluetooth and sends the text commands to the Bluetooth module. This module is connected to a microchip controller of the PIC16F877A family. This controller interprets the commands and performs the appropriate actions. The control of electrical circuits is done with a separated system, to isolate the load from the control circuitry. The system also sends back feedback to alert the user about the result of the command. This system's voice command feature makes it universally accessible. However the usage of SMS makes it unreliable. Also the necessity of two phones, one with the user and another in proximity to the controller can lead to additional expenses.

A system ${ }^{[7]}$ uses the GSM network along with an AVR microcontroller. This is also an SMS based system. The user enters the commands. These are sent via SMS. However, this system uses a standardized AVR code that can be easily interpreted by the microcontroller. There is a GSM module that is attached to the AVR. This will receive the commands that are sent via SMS. AT commands are used to communicate with the modem. The AVR in turn instructs a driver circuit to control the appliances as necessary. This system has remote access capabilities from all over the world. However it cannot function in real time.

A PIC16F887 microcontroller along with GSM ${ }^{[8]}$ can form a home automation system. The commands are sent from the mobile phone via GSM to the GSM modem. This command is converted to text and sent to the controller through a RS-232 bus. These commands are interpreted by the microcontroller and the corresponding action is performed. The drawback of this system is that it requires external power supply. Also, it cannot control multiple appliances concurrently. The system cannot verify the status of the appliances at a time. 


\subsection{Bluetooth Based Home Automation}

The system shown in figure 2 makes use of a cell phone and Bluetooth technology ${ }^{[9]}$. Bluetooth technology is secured and low cost. It makes use of an Arduino Bluetooth board. An interactive python program is used in the cell phone to provide the user interface. The I/O ports of the Bluetooth board and relays are used for interfacing with the devices which are to be controlled. The Bluetooth is password protected to ensure that the system is secure and not misused by any intruders. The Bluetooth has a range of 10 to 100 meters, $2.4 \mathrm{GHz}$ bandwidth and 3Mbps speed. The python app on the phone is portable. It is also a fast and cost effective system. There is a diagnostic system that can detect problems in the circuitry. A feedback system will report status of devices after every signal toggle. The main drawback with respect to Bluetooth is that it takes a long time to discover and access devices in its vicinity. It does not provide energy conservation tips. Real time access cannot be achieved. Anywhere access to the devices cannot be achieved. Access is limited to within the Bluetooth range.

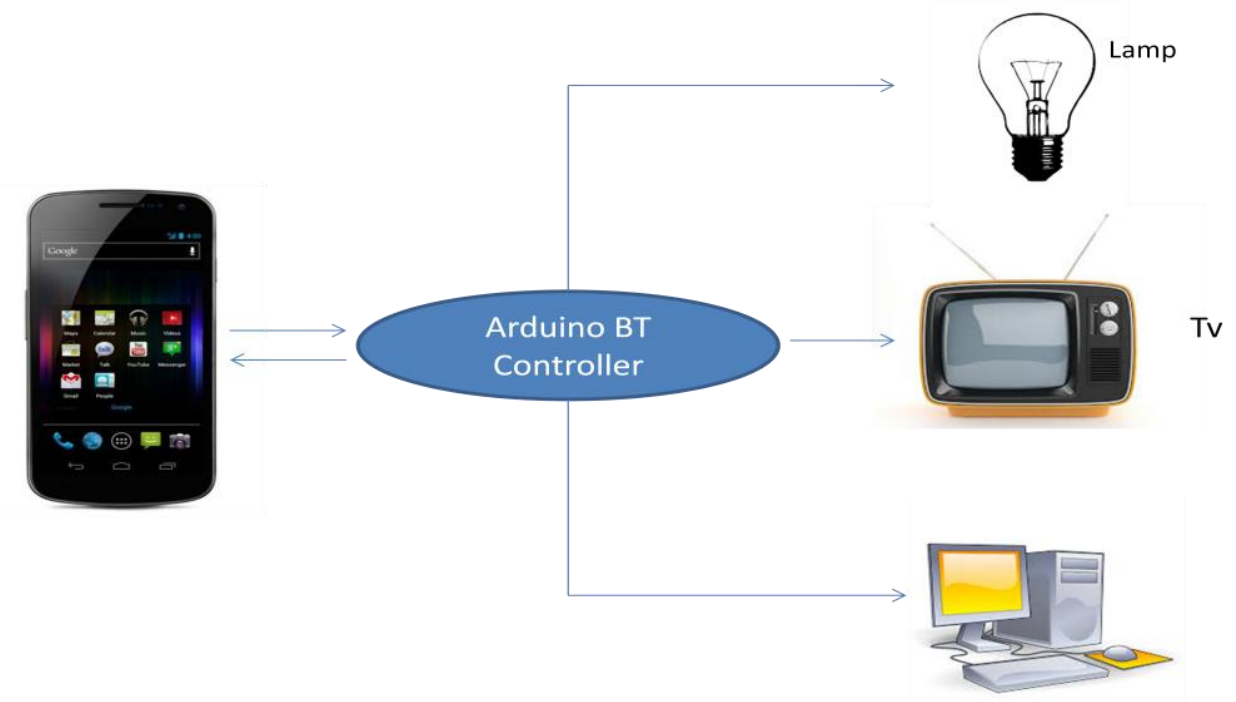

Figure 2 Block Diagram of Home Automation

Bluetooth technology is used to control home appliances ${ }^{[10]}$. The client is a PC that is connected via USB to the Bluetooth module, sensor circuit and a pulse width modulation circuit. Sensors and actuators are used to control the circuit. The Bluetooth module that is connected to it will allow it to receive various commands via Bluetooth. Bluetooth devices can scan and detect other devices easily. It might also be possible to check whether devices are working properly or not. The system also has an illumination sensor that can turn on lights when external light is dull and a temperature sensor. This system also suffers from the drawback of the range of Bluetooth being around 10 meters only. This system has the advantage of being able to fit onto an existing system. There is also low cost involved in this system.

\subsection{Phone Based Home Automation}

Some systems ${ }^{[11]}$ are described as an enabling system that can be used to provide a common framework for home Automation. It provides a system for a smart home that includes facilities such as a system controller, housewide wiring and a common interface. This will enable using the existing system for home automation.

A hardware based remote controller for power point control has been described ${ }^{[12]}$. The function of this remote controller is to control the power supplied to devices at a remote location. The system uses the telephone line for transmitting the commands. The controller is a logic system built entirely of hardware. It eliminates the cost incurred with microcontrollers. It uses a DTMF transceiver which is interfaced with a solid state relay to control the power supply. It could also be implemented experimentally with infra red signals and AC power line carrier technology.
A home automation system makes use of the dual tone multi frequency (DTMF) used in telephone lines ${ }^{[13]}$. The system as shown in figure 3 uses the standard public-switched telephone lines. There are three components in the system. The first is the DTMF receiver and ring detector. The second part is the IO interface unit. The third part is the PC which does the online operations. The PC detects the ringing of the line and then authenticates the user. After this the user will be allowed to use the keypad tones to control the devices as required. An example of stepper motor control is taken up. This system has the advantage of being secure and allowing international standardization. This is because the DTMF tones are the same all over the world. But it suffers from the drawback that the number of appliances is limited by the number of keys in the keypad. An ordinary phone usually has 12 keys only. 


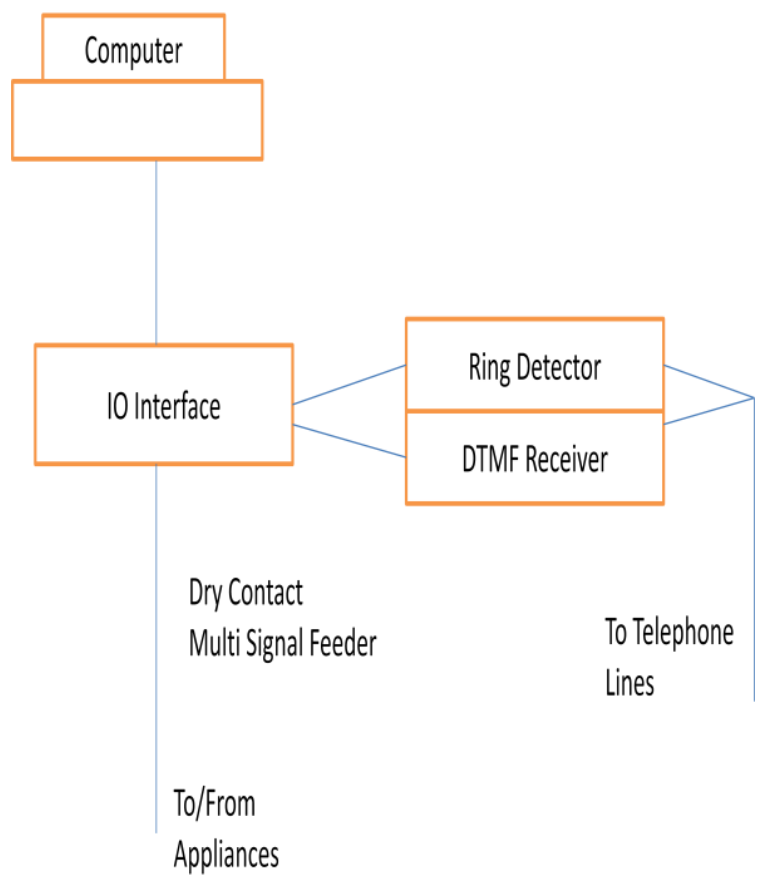

Figure 3 Smart Control System to control Appliances remotely by phone

\subsection{ZigBee Based Home Automation}

The ZigBee wireless communication technology can be applied ${ }^{[14]}$ for home automation. The system uses PIC microcontroller and voice recognition for this purpose. The voice commands are taken from a mike. They are compared with a voice store and processed. The PIC microcontroller then transmits the commands through ZigBee to the receiver. The receiver unit has another PIC microcontroller that can process the command. It uses relays to control the respective appliances. This system has the drawback that ZigBee is a low range communication medium. So remote access is hindered from faraway locations. Also, the voice recognition module could become unwieldy. This system has the added feature of integrating a smoke detector to the system. When smoke is sensed, it sends a message to the user's built-in mobile number.

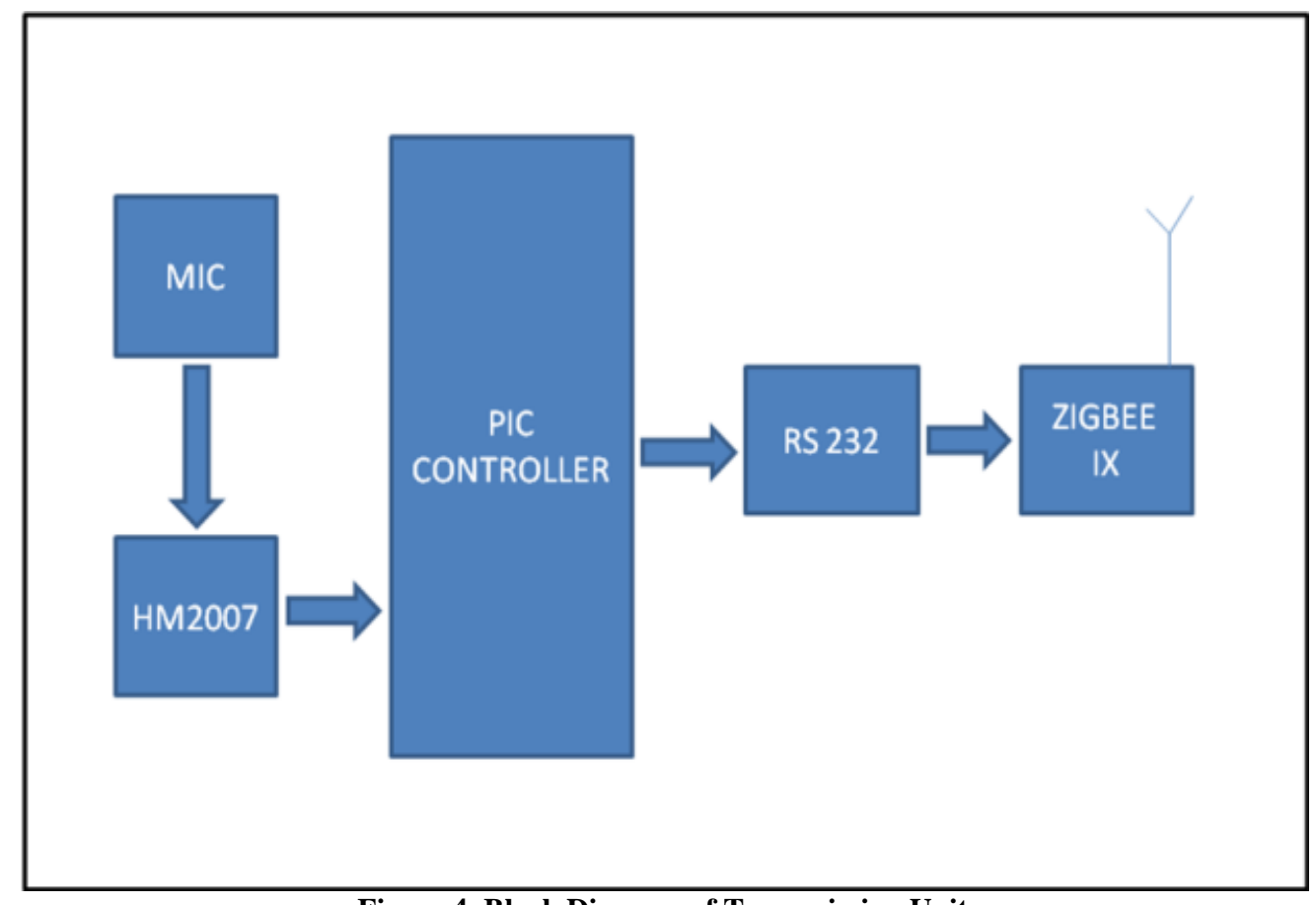

Figure 4. Block Diagram of Transmission Unit

\subsection{Wireless Control Systems}

Systems using wireless communication can be made ${ }^{[15]}$ by linking up stand alone appliances that are present at home or in office and integrating to form a co operating network. A combination of various technologies like $\mathrm{Wi}-\mathrm{Fi}$ and Bluetooth are used to integrate the system. Such a system is laid out as illustrated in figure $5 \& 6$. The universal Plug and play capability is used to provide a transparent network of devices to the user. The system makes use of the Open Service Gateway Interface (OSGi). The appliances are connected via different networking technologies. The user application layer makes use of web browsers, pocket PC application and a central console. Speech based commands can also be used for controlling the appliances. Advanced features are provided such as device discovery and device connection. The entire system is implemented in a Linux platform. The system also has the ability to add intelligent control modules. These control modules are capable of knowledge capturing and pattern recognition. The universal plug and play system uses many standard protocols for interoperability. The main advantage of the system is its interoperability. Another advantage is the dynamic discovery of the service. It also has the ability for sharing of service. 


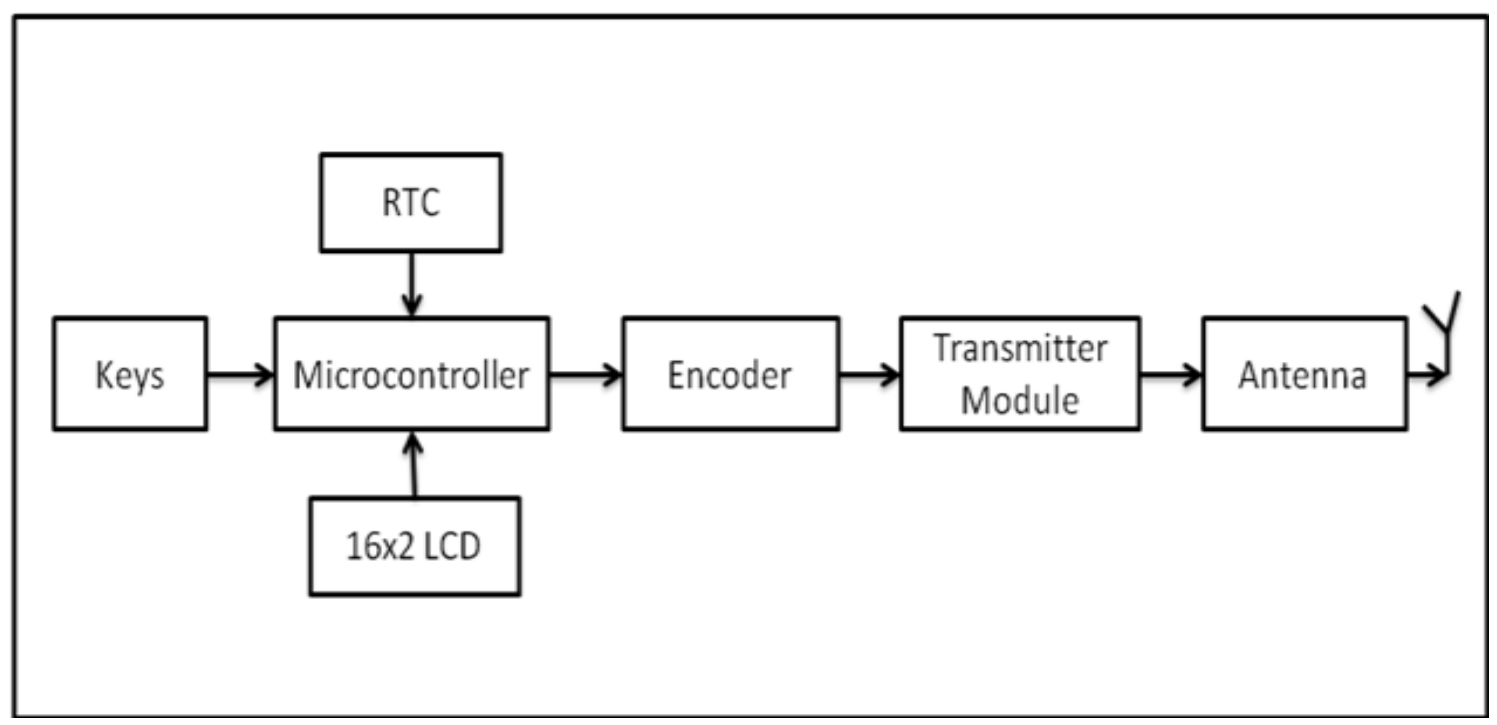

Figure 5 Block Diagram of Transmitter Section

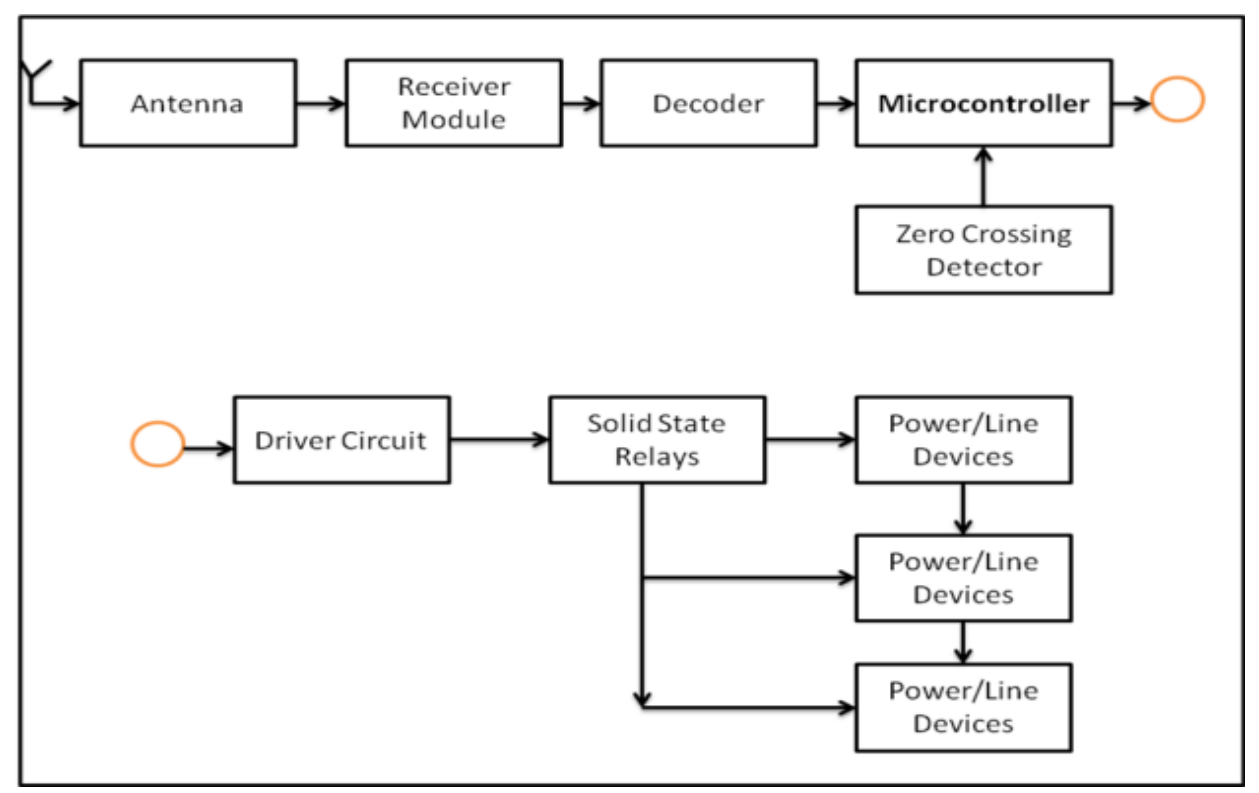

Figure 6 Block Diagram of a receiver Section

The system proposed uses an Arduino board along with Wi-Fi communication technology ${ }^{[16]}$. It also has the option of another automated mode where there are temperature and light sensors which can be set to control the appliances depending on the conditions. The system uses an Android smart phone to communicate with a home PC those servers as a server. The PC is connected to and controls the Arduino board. The GUI is designed using MATLAB. The mobile phone can communicate with the PC through Internet. Internet access is through Wi-Fi. However, this extension is not implemented in the system. The system has the advantage of remote access through the phone. The drawback is that the PC needs to be left on all the time. Also it depends on Wi-Fi for internet access which is not reliable and not widely available.

\subsection{Mixed Type}

Home automation is achieved ${ }^{[17]}$ with a combination of GSM, Bluetooth and ZigBee technologies. The user interfacing is done through an Android application. This application takes user voice input and maps it to a set of textual commands. These commands are sent through SMS to another phone at home. This in turn transfers the commands through Bluetooth to a PIC controller. This is called a remote unit. This remote unit will then send the commands to a ZigBee transceiver. This transfers these commands through ZigBee to the main controller. The controller interprets the commands and performs the required operations. It also sends back status information through ZigBee. This system is usable and can offer remote access. However, the system can become expensive due the use of multiple controllers and technologies. Also, the SMS system can prove to be unreliable.

A study of the various technologies that can be applied to home automation is done ${ }^{[18]}$. Specifically, it talks about how modern day networking protocols can be applied to enable home automation. It proposes that standardization of home automation technologies can be achieved using Internet Protocol (IP). The UPnP is an extension of the plug and play capabilities. The paper recommends use of GSM networks as a backup facility, while a web based application can be the primary user interface. Technologies like Wi-Fi and X-10 protocols can be used to ensure connectivity with the home 
network. It also emphasizes the role of a good UI. The system is illustrated in figure 7.

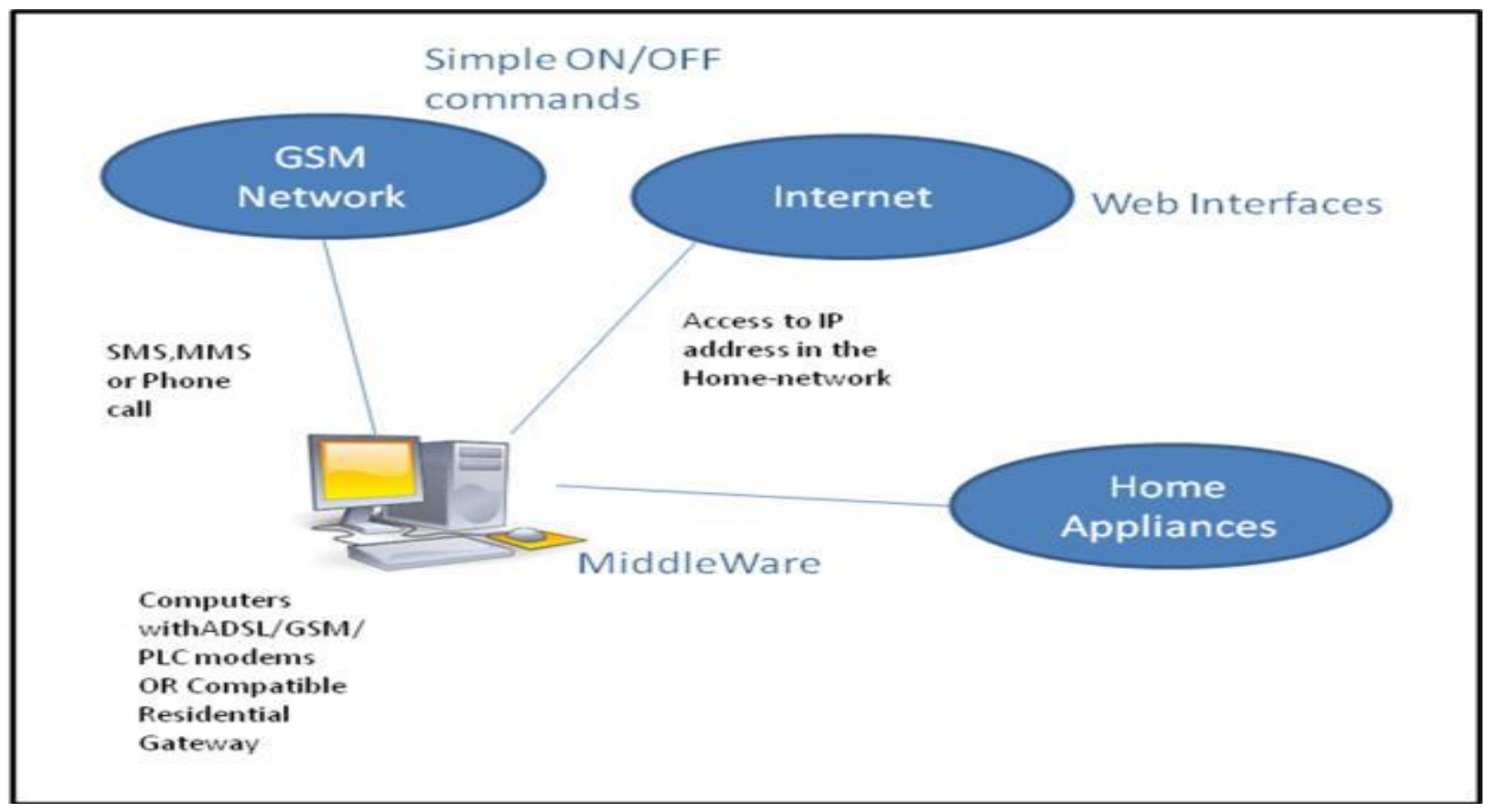

Figure 7 Remote-Controlled HAS

An alternate solutions for home automation ${ }^{[19]}$ combines GSM and ZigBee. It uses SMS to send commands from the remote mobile to the home mobile. In case of suspect activity, the system can make use of GSM to send SMS alert or automated calls to the user. By replying the user can take appropriate measures. The hardware is implemented using

ZigBee EM357 module, Atmega128 MCU (microcontroller unit) and Sony Ericsson T290i mobile phone set. The MCU will deal with both the ZigBee and GSM networks. The sensors communicate with the MCU using the ZigBee system. The entire system can be implemented using $C$ language. The relay controls the home appliances. The relays are isolated from the control section due to the presence of the ZigBee network. The advantage of the system is its low cost and user friendliness. It also offers home security which is an added feature. However, it is not a real time system.

Many non conventional systems for home automation ${ }^{[20]}$ are available. A combination of GSM along with a FPGA (Field Programmable Gate Array) system. This is due to the flexibility provided by the FPGA which can be programmed to suit the users' needs. It is also less expensive compared to a microcontroller. This helps to decrease the price of such systems. The FPGA is interfaced with a GSM modem. The modem is used to communicate with the devices. The FPGA makes the decisions regarding the control of devices. A UART is used to allow the interfacing between the GSM modem and the FPGA. The advantage of such a system is that they can be used as real time systems. They offer security as they are hard wired and cannot be attacked by malicious intent. They can also add or subtract features as required by the user.

\section{DISCUSSION}

This paper also gives a comparison of all the above systems described. The systems that have been studied have certain common features. All these systems use a basic underlying communications technology. The advantages and drawbacks of the system derive from this underlying technology. All the systems have a control circuitry that is used to interface withthe electrical appliances. There has to be a common command system that will be used to issue commands to the control circuits. The next important feature of the system is the user interface. This determines how the user will interact with the system and extent of control the user exerts over the system. This influences the usability of the system. Most systems also have security features to ensure only authorized access.

\section{Table 1 Consolidated Comparison Report of all System}

\begin{tabular}{|c|c|c|c|c|c|c|}
\hline System & $\begin{array}{c}\text { Primary } \\
\text { Communication }\end{array}$ & Remote access & $\begin{array}{c}\text { Number of } \\
\text { Devices }\end{array}$ & Cost & Speed & Real Time \\
\hline GSM & SMS messages & $\begin{array}{c}\text { Access from } \\
\text { anywhere in the } \\
\text { world }\end{array}$ & Unlimited & $\begin{array}{c}\text { High cost due } \\
\text { to SMS charges }\end{array}$ & $\begin{array}{c}\text { Slow due to } \\
\text { delivery issues }\end{array}$ & No \\
\hline Bluetooth & $\begin{array}{c}\text { Bluetooth and } \\
\text { AT commands }\end{array}$ & $\begin{array}{c}\text { Restricted to } \\
\text { Bluetooth } \\
\text { range- 10 } \\
\text { metres }\end{array}$ & Unlimited & $\begin{array}{c}\text { Fast due to } \\
\text { proximity }\end{array}$ & $\begin{array}{c}\text { Fast due to } \\
\text { proximity }\end{array}$ & Yes \\
\hline
\end{tabular}




\begin{tabular}{|c|c|c|c|c|c|c|}
\hline Phone Based & Phone lines & $\begin{array}{c}\text { Anywhere with } \\
\text { a phone line } \\
\text { Zigbee }\end{array}$ & $\begin{array}{c}12 \text { due to 12 } \\
\text { frequencies of } \\
\text { DTMF }\end{array}$ & Fast & Fast & Fo \\
\hline Wireless & $\begin{array}{c}\text { Radio, infrared } \\
\text { or other waves }\end{array}$ & $\begin{array}{c}\text { Depending on } \\
\text { range and } \\
\text { spectrum of } \\
\text { waves used }\end{array}$ & Unlimited & $\begin{array}{c}\text { High cost due } \\
\text { to licensing and } \\
\text { other spectrum } \\
\text { issues }\end{array}$ & $\begin{array}{c}\text { Slow due to } \\
\text { interferences }\end{array}$ & Yes \\
\hline
\end{tabular}

The most common types of methodologies seen from the above surveyed systems are GSM, Bluetooth, based, wireless and combinations of these.

GSM systems primarily use SMS messages to communicate the commands issued by the user to the main control system at home. This system offers the ability to control the appliances from all over the world. However, the cost incurred can be large depending on the location. Another significant drawback is that the system lacks reliability. There is no assurance of the delivery of the message to the system. Thus such a system cannot be used as a real time system. GSM can be used as a method of Internet access and a web application can be used as a method of user interfacing.

A Bluetooth system may use either a mobile phone or a PC as the receiver. The Bluetooth system can offer comprehensive control of the home appliances as long as the user is at home. It can function as a real time system. The speed of communication is high. This means that the user can be alerted about events as and when required. There is also greater security in Bluetooth technology. However, it cannot offer control when outside home. The range of Bluetooth appliances is around 10 meters. This is a significant drawback of this system.

Phone based systems can use the dual tone multiple frequency to transmit commands. This system depends on the ability to make phone calls from a remote location to a phone line at home. This has the advantage of offering remote access from anywhere in the world from where they can make a call. This can offer an almost real-time system. The drawback here is that it limits the number of possible devices to the number of possible DTMF tones. Also, it is hard for the system to give feedback to the user.

ZigBee is an alternate technology that is similar to Bluetooth. This has the same advantages and drawbacks as the Bluetooth based system. This is a fairly new technology.

Wireless automation systems can use a host of wireless communication techniques. This may be radio frequency waves or infrared waves. This can also be used to power a real time system. The only downside her is the range and availability of the spectrum. Radio waves have a much larger range and offer good options for remote access. However the availability of the spectrum has to be taken into account.

Certain bands of the spectrum are in demand while the unlicensed bands are used by many other applications. There is the possibility of interference. This compromises the security of the system.

Many systems exist that use a combination of the methodologies to compensate for the drawbacks of each. Such a composite implementation can lead to sound systems. The only thing that may affect such systems is the cost of the systems and the possibility of redundancy.
The user interface is another aspect in which systems vary widely. Early systems used minimal UI or had no UI and used keypads for the user to enter commands to the controllers. But modern systems have intuitive UIs and a lot of care is taken in designing them. Android smart phones are the most popular choice. They have a wide variety of tools allowing easy development. Smart phones in general are a handy tool for controlling home automation systems. Another option is through web applications which could run in browsers. This is also a popular choice, but not as handy. Refer to Table 1 for a consolidated comparison of all the systems.

\section{CONCLUSION}

Based on all the systems surveyed and their advantages and drawbacks, this paper presents the features to be possessed by an ideal system for home automation with remote access. An ideal system should be available from all over the world to a user and in real time. A GSM network is identified as a candidate for this. However, the data channel of GSM must be used, to provide internet access. Only the Internet can ensure that access can be made available at all times. This will give rise to a standard access method for the home appliances using the Internet protocol. The user interface should be a web application that has an associated mobile application. So that people of all kinds can access the system. Such a system should also have the feature of being easy to install. Only then can automated homes become commercially viable. There should be a lot of thought put into the design of the user interface for these apps. Plug and play capabilities will be an added bonus for the system. Ease of adding a new device to an automated house will play an important role in taking forward the systems commercially.

Future scope for the home automation systems involves making homes even smarter. Homes can be interfaced with sensors including motion sensors, light sensors and temperature sensors and provide automated toggling of devices based on conditions. More energy can be conserved by ensuring occupation of the house before turning on devices and checking brightness and turning off lights if not necessary. The system can be integrated closely with home security solutions to allow greater control and safety for home owners. The next step would be to extend this system to automate a large scale environment, such as offices and factories.

\section{REFERENCES}

[1] Baris Yuksekkaya, A. Alper Kayalar, M. Bilgehan Tosun, M. Kaan Ozcan, and Ali Ziya Alkar "A GSM, Internet and Speech Controlled Wireless Interactive Home Automation System", 2006, IEEE Transactions on Consumer Electronics, Vol. 52(3), pp. 837 - 843.

[2] Rozita Teymourzadeh,Salah Addin Ahmed,Kok Wai Chan and Mok Vee Hoong, "Smart GSM Based Home Automation System", 2013, IEEE Conference on Systems, Process \& Control, Kuala Lumpur, Malaysia. 
[3] A. Alheraish, "Design and Implementation of Home Automation System", 2004, IEEE Transactions on Consumer Electronics, Vol. 50(4), pp. 1087-1092.

[4] M.Van Der Werff, X. Gui and W.L. Xu, "A Mobile based Home Automation System, Applications and Systems", 2005, 2nd International Conference on Mobile Technology, Guangzhou, pp.5.

[5] Mahesh.N.Jivani, "GSM Based Home Automation System Using App-Inventor for Android Mobile Phone", 2014, International Journal of Advanced Research in Electrical, Electronics and Instrumentation Engineering, Vol. 3(9), pp. 12121-12128.

[6] Faisal Baig, Saira Baig, Muhammad Fahad Khan, "Controlling Home Appliance Remotely through Voice Command", 2012, International Journal of Computer Applications, Vol. 48(17), pp.1 - 5.

[7] S.R.Bharanialankar, C.S.Manikanda Babu, "Intelligent HomeApliance Status Intimation Control and System Using GSM", 2014, International Journal of Advanced Research in Computer Science and Software Engineering, Vol. 4(4), pp.554 - 556.

[8] Rozita Teymourzadef, Salah Addin Ahmed, Kok Wai Chan, Mok Vee Hoong, "Smart GSM Based Home Automation System", 2013, IEEE Conference on Systems, Process \& Control, Kuala Lumpur, Malaysia, pp.306 - 309.

[9] R.Piyare,M.Tazil, " Bluetooth Based Home Automation System Using Cell Phone”, 2011 ,IEEE $15^{\text {th }}$ International Symposium on Consumer Electronics, Singapore, pp. $192-195$.

[10] Home Automation System via Bluetooth Home Network", 2003, SICE Annual Conference, Fukui, Vol. 3, pp. $2824-2829$.

[11] H. Brooke Stauffer "Smart Enabling System for Home automation", 1991, IEEE Transactions on Consumer Electronics, Vol. 37(2) , pp. 29-35.

[12] Eddie M C Wong, "A Phone Based Remote Controller for Home and Office Automation", 1994, IEEE Transactions on Consumer Electronics, Vol. 40(1), pp. 28-34.
[13] Baki Koyuncu, "PC Remote Control of Appliances by Using Telephone Lines", 1995, IEEE Transactions on Consumer Electronics, Vol. 41(1), pp. 201-209.

[14] V.Sathya Narayanan, S.Gayathri, "Design of Wireless Home Automation and security system using PIC Microcontroller", 2013, International Journal of Computer Applications in Engineering Sciences, Vol. 3 ( Special Issue), pp. 135- 140.

[15] Baris Yuksekkaya, A. Alper Kayalar, M. Bilgehan Tosun, M. Kaan Ozcan and Ali Ziya Alkar, "A GSM, Internet and Speech Controlled Wireless Interactive Home Automation System", 2006, IEEE Transactions on Consumer Electronics, Vol. 52(3), pp. 837-843.

[16] Bader M. O. Al-thobaiti, Iman I. M. Abosolaiman, Mahdi H. M. Alzaharani, Sami H. . Almalki, Mohamed S. Soliman, "Design and Implementation of a Reliable Wireless Real-Time Home Automation System Based on Arduino Uno Single-Board Microcontroller", 2014, International Journal of Control, Automation and Systems, Vol. 3(3), pp. $11-15$.

[17] Faisal Baig, Saira Beg and Muhammad Fahad Khan, "ZigBee Based Home Appliances Controlling Through Spoken Commands Using Handheld Devices", 2013, International Journal of Smart Home, Vol. 7(1), pp 19 -26 .

[18] Armando Roy Delgado, Rich Picking, Vic Grout, "Remote-Controlled Home Automation Systems with Different Network Technologies", Centre for Applied Internet Research, University of Wales, UK.

[19] Arnab Waheed Ahmad, Naeem Jan, Asaeed Iqbal, Chankil Lee, Ansan Korea, "Implementation of ZigBeeGSM based Home Security Monitoring and Remote Control System", 2011, IEEE Symposium on Circuits and Systems (MWSCAS), Seoul, pp. 1-4.

[20] Pooja S Chinchansure, Charudatta V Kulkarni, "Home Automation System based on FPGA and GSM", 2014, International Conference on Computer Communication and Informatics (ICCCI), Coimbatore, India, pp. 1 - 5 